\title{
Cow's milk exclusion in young children: Parents motives, sources of information and perception of their child's eating behaviours
}

\section{Abstract}

Research has assessed the diets of young children that follow a diet that excludes cow's milk. However, there is insufficient information about why parents decide to exclude cow's milk, where they obtain nutritional information, and their child's eating behaviours. This is important as the prevalence of cow's milk allergy is increasingly common in children, alongside a growing preference for plantbased diets. The purpose of this research was firstly to explore why parents exclude cow's milk from their child's diets and if this is due to recommendations from a health care professional. Secondly, to understand where parents source information to enable them to provide a healthy diet for their child. Finally, to explore parent's opinions of their child's eating behaviours.

Thirty parents of children aged 1-3 years who exclude cow's milk and 20 parents of children who did not follow a restricted diet completed an online questionnaire. The questionnaire comprised of three blocks of questions. The first asked about the parents, the second pertained to the child. If they excluded cow's milk they were asked why they made this decision and who advised them. Parents were also asked where they source nutrition information for their child. The final block of questions consisted of the Child Eating Behaviour Questionnaire.

Parents reported excluding cow's milk due to cow's milk allergy $(60 \%)$, lactose or milk intolerance $(10 \%)$, suspected allergy or intolerance $(27 \%)$ or personal choice $(3 \%)$. When asked "Who advised you to remove cow's milk from your child's diet?" the majority responded that it was their own decision (35\%), followed by GP advice $(24 \%)$. The most common sources of nutrition information for parents of children that exclude cow's milk came from parenting webpages $(21 \%)$, and social media (19\%), whilst those on an unrestricted diet obtained information from health visitors $(24 \%)$ and parenting webpages $(19 \%)$. There were no differences in eating behaviours between children that excluded cow's milk and children that did not except for satiety responsiveness $(p=0.014)$, which was greater in the cow's milk exclusion group.

This preliminary data indicates that many parents exclude cow's milk from their child's diets based on their own advice. Where parents of children on an unrestricted diet sourced nutrition information from their health visitor, few parents of children who exclude cow's milk used this source of support $(7 \%)$.

\section{Conflict of Interest}

There is no conflict of interest 